standard form. Similar approximations may be obtained for the higher-order functions $t^{n} I_{R}{ }^{(n)}$, but none will be presented here since these functions can be computed about as easily by Gauss-Laguerre quadrature, as previously described.

6. Acknowledgment. I should like to thank Dr. R. P. Kenan for his assistance and suggestions concerning ('omputer programming and other aspects of this work.

Battelle Memorial Institute

Columbus, Ohio

1. H. S. Carsla $\&$ J. C. Jaeger, Conduction of Heat in Solids, 2nd ed., Oxford Univ. Press, New York, 1959, pp. 339-341.

2. R. H. Ritchie \& A. Y. SaKaklra, "Asymptotic expansions of solutions of the heat conduction equation in internally bounded cylindrical geometry," J. A ppl. Phys., v. 27, 1956, pp. $1453-1459$. MR 18, 780.

3. L. V. Spencer \& U. Fano, Phys. Rev., v. 93, 1954, pp. 1172-1181.

4. (․ H. Hardy, Ramanujan, Cambridge Univ. Press, Cambridge; Macmillan, New York, 1940, pp. 195-198. MR 2, 210.

5. V. Volterra, Atti. Accad. Lincei Mem. Cl. Sci. Fiz. Mat. Nat., v. 11, 1916, pp. 167-249.

6. A. Erdélyi, W. Magnus, F. Oberhettinger \& F. G. Tricomi, Higher Transcendental Functions, Vol. III, McGraw-Hill, New York, 1955, pp. 217-224. MR 16, 586.

7. A. Erdélyi, W. Magnus, F. Oberhettinger \& F. G. Tricomi, Higher T'ranscendental Functions, Vol. I, Me(iraw-Hill, New York, 1953, p. 266. MR 15, 419.

8. P. R A B INOWITZ \& G. WEISS, "Tables of abscissas and weights for numerical evaluation of integrals of the form $\int_{0}^{\infty} e^{-x} x^{n} f(x) d x$, "MTAC, v. 13, 1959, pp. 285-294. MR $21 \# 6713$.

9. J. STOER, "A direct method for Chebyshev approximation by rational functions," J. Assoc. Comput. Mach., v. 11, 1964, pp. 59-69. MR 29 \#1479.

\title{
Note on the Calculation of Fourier Series
}

\section{By Philip Rudnick}

Cooley and Tukey have recently presented an algorithm for the machine calculation of Fourier series [1]. In this connection mention should be made of the similar method described by Danielson and Lanczos [2]. Although the latter is less elegant and is phrased wholly in terms of real quantities, it yields the same results as the binary form of the Cooley-Tukey algorithm with a comparable number of arithmetical operations.

A small-computer program has been written in this laboratory which uses the Danielson-Lanczos method with one minor modification, described below. ${ }^{*}$ In this form cosine and sine series may be evaluated independently of one another, and as with the Cooley-Tukey process, the calculation can be performed by replacing input data with results, without any substantial storage requirement beyond that set by the original number of input coefficients. The procedure used is readily extensible to the computation of $[(N / 2)+1]$ cosine and $[(N / 2)-1]$ sine sums, from an original sequence of $N$ real numbers, where $N$ is any power of 2 greater than the fourth. Two executions of this procedure will yield values for a set of $N$ complex Fourier series of $N$ terms each, and can be accomplished by $N\left[\log _{2} N+3\right]$ real multiplications and $7 N / 2\left[\log _{2} N-23 / 7\right]$ real additions. For the same task the

Received December 29, 196:).

* Work of the laboratory is sponsored by the Office of Naval Research. 
indicated requirement of the Cooley-Tukey algorithm is approximately $2 N \log _{2} N$ each of complex additions and complex multiplications. $\dagger$

Modification of the Danielson-Lanczos algorithm. Three equations given by Danielson and Lanczos [2] on pages 378 and 379 may be replaced by the following likenumbered equations.

$$
\begin{aligned}
\bar{u}_{0} & =u_{1}, \\
\bar{u}_{i} & =u_{2 i-1}+u_{2 i+1}, \quad 1<i<2 n, \\
\bar{u}_{2 n} & =u_{4 n-1}, \\
\bar{v}_{i} & =v_{2 i-1}+v_{2 i+1}, \quad 1<i<2 n \\
A_{k}{ }^{\prime} & =\frac{1}{2} \sec (\pi k / 4 n) \sum_{\alpha=0}^{2 n} \bar{u}_{\alpha} \cos (\pi k \alpha / 2 n), \quad 0 \leqq k<2 n, \\
A_{2 n}^{\prime} & =0 \\
B_{k}{ }^{\prime} & =\frac{1}{2} \sec (\pi k / 4 n) \sum_{\alpha=1}^{2 n-1} \bar{v}_{\alpha} \sin (\pi k \alpha / 2 n), \quad 0<k<2 n, \\
B_{2 n}^{\prime} & =\sum_{\alpha=0}^{2 n-1}(-1)^{\alpha} v_{2 \alpha+1} .
\end{aligned}
$$

The original equation (53) is in error; the low limit on the summation index should be 0 , consistent with (58) above.

With this modification the cosine and sine series calculations are more closely alike (except for the second equation (58)), and are independent of one another.

University of California, San Diego

Marine Physical Laboratory of the

Scripps Institution of Oceanography

San Diego, California 92152

1. J. W. Cooley \& J. W. Tukey, "An algorithm for the machine calculation of complex Fourier series," Math.Comp.,v. 19, 1965, pp. 297-301. MR 31 \#2843.

2. G. C. DANIELSON \& C. Lanczos, "Some improvements in practical Fourier analysis and their application to X-ray scattering from liquids," J. Franklin Inst., v. 233, 1942, pp. $365-380 ; 435-452$. MR 3, 276.

† I am indebted to a private communication from the referee for the information that this expression, interpreted on the basis that one complex addition and multiplication requires four each of real additions and real multiplications, considerably understates the efficiency of programs which have been written based on the Cooley-Tukey algorithm. 